\title{
Merge After Toll
}

\section{Jiamei Guo,Xianjing Chen,Shuwen Yangming,Jiangying Chen*}

Zhejiang Province Ningbo University,Ningbo Zhejiang 315000

\author{
Key Words: Fluid Mechanics Theory, Cellular Automaton, Security Issues, Minimum Fan Toll Plaza \\ Design
}

\begin{abstract}
In order to design a reasonable toll station fan in area, we regard the flow as an incompressible fluid approximately. Combine the relationship between vehicle density and velocity under the smallest safety distance with the above characteristics, we can obtain the parameter equation between the width and the length of the fan in area. The merging pattern has three modes. They are oppression lane changing, free lane changing and other car oppression lane changing. We mainly analyze the situation of oppression lane changing. We take the collision probability as a limitation factor to draw the shape of the fan in area. Combining with the driver's reaction time of automatic driving and manual driving and three kinds of tollbooths' service time, we can improve the width of the fan in area. According to the proportion of automatic vehicles, we can obtain the tollbooths' the best placement program.According to the toll station design specifications, we determine the design of the fan-in area is combined with a straight road and a trapezoidal platform. In order to analyze the safety performance of fan-in area, we have established a matrix about the desire and delimit the probability of the vehicle collides to description.
\end{abstract}

\section{Introduction}

Highway toll station will pass the two ways "ramp fees" and "barrier tolls" to charge the driver's high-speed fees. This charging method's window usually is more than article lane number. Therefore, when the vehicles drive out of toll station, they must "fan in" from the larger number of tollbooth egress lanes to the smaller number of regular travel lanes. Toll plaza is established for improving traffic congestion, including the fan-out area before the barrier toll, toll station itself and area of the fan in after toll station.

\section{Modeling the Design of the Tollbooth}

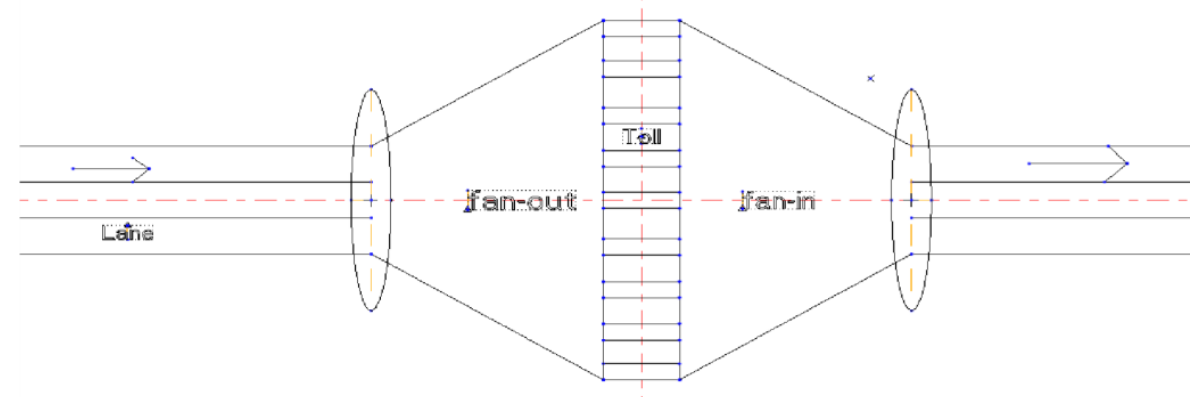

Figure1: The top view of a toll plaza.

The model is mainly established on the "Fan-in" area. Vehicles go through the tollbooth to the lane with a constant acceleration a. We regard the vehicle driving process in the Fan-in area as the incompressible fluid flow in the container. If the incompressible fluid's density change can be ignored in the process of flow, we call this flow as incompressible fluid. And in the process of driving, the vehicles' surface density can be regarded as a amount that can be controlled artificially. When the flow is constant, with the increase of the speed of the vehicle, the vehicle distance between each vehicle will increase and the vehicles' surface density will reduce. 
The definition of flow refers to the amount of fluid flowing through the section in unit time. When the volume of fluid is represented by volume, the volume of fluid flowing through a section of pipe in a unit time is called the volume flow of the cross section. It's denoted as [1]:

$$
Q=\rho^{\prime} v^{\prime}
$$

$\rho^{\prime}$ isthe density of fluid itself and $v^{\prime}$ is the speed of the fluid through the cross section. To fit the fluid flowing model into traffic model, equationcan be modified to:

$$
Q=\rho w v
$$

Where stand by traffic flow and $v$ is the vehicle's velocity. $\rho$ is the surface density of vehicles which means the number of vehicles in the unit length along the flow direction for every lanes, and $w$ is the width of the fan in the area.

\section{Model Analyzing and Solving}

According to equation,we can get

$$
w=\frac{Q}{\rho v}
$$

Using this equation, we can calculate the width of the fan in the area.

Assuming that in a crowded condition, there are $\mathrm{n}$ vehicles on some way. The vehicle $i$ has a speed $v_{i}$ and its maximum acceleration is $a_{i}$ at a certain moment. The length of the vehicle is $y_{i}$ and the total length of the road is $Y$. The minimum distance between the vehicle $i$ and vehiclei-1is $d_{i-1}$.According to the reference, the following formula is derived [2]

$$
d_{i-1}={ }_{i} t+\frac{v_{i}}{2 a_{i}}-\frac{v_{i}^{2}}{2 a_{i-1}}
$$

The road traffic density $\rho$ :

$$
\rho=\frac{n}{\sum_{i=1}^{n} y_{i}+\sum_{i=1}^{n-1} d_{i}}
$$

In the same circumstances $v_{n}$ and $v_{1}$ are approximately equal. And $a_{n}$ and $a_{1}$ are approximately equal. Thus, we can get:

$$
\rho=\frac{1}{t_{r}(v-c)+(l+g)}
$$

From the above type we can know the relationship between the speed and the width of the fan in area when the vehicle flow is certain.

$$
w=\frac{Q_{v}\left[t_{r}(v-c)+(y+g)\right]}{v}
$$

Due to the constant acceleration of the vehicle, if the speed of the vehicle is increasing, the width of the fan area is reduced.

When the number of toll stations does not change, if increase the number of lanes, the difference of numbers between the toll stations and the lanes decrease. We can see the comparison from the above image and data. When the number of lanes and toll stations are closing. Although the fan in area of the overshoot section is reduced, thecorner's traffic at the maximum corner is very non-smooth. The probability of traffic accidents is very high.

When the number of lanes does not change, if increase the number of toll stations, the difference of numbers between the toll stations and the lanes increase. When the number of lanes and toll stations are not closing. Although the fan in area of the overshoot section is increased, the corner's traffic at the maximum corner is very smooth. The probability of traffic accidents is lower.

Empirically, the fan in and fan out area are symmetrical to each other. So their surface areas are the same. 


\section{Merging Pattern}

The number of lanes decreases as the vehicle moving from the fan in area to the highway. If all vehicles go out of the tollbooths at the same time, some vehicles need to change lanes. There are three modes to change line. They are oppression lane changing, free lane changing and other car oppression lane changing.

Oppression lane change means restricting the number of lanes, the vehicle had to change, even though a lot of vehicles, or it will hit the "wall”. Free lane change means the driver randomly changes lanes. Other car oppression lane change means by the impact of other vehicles changing lanes, the driver has to change lanes.

It's difficult for us to calculate the first two cases.Therefore, we only consider the pressure lane change situation. When the car changing the lanes, if the distance between the two vehicles is less than or equal to the length of the vehicle, the vehicles will collide. Let symbolize " $A$ " denote the event of two cars collision and " $L_{i}$ " denote cars are on thei-thlane, thus, using total probability formula:

$$
P(A)=\sum_{i=1}^{n} P\left(L_{i}\right) P\left(A \mid L_{i}\right)
$$

Where n denote the number of the lanes. $P\left(L_{i}\right)$ meansthe probability of the vehicle drive to the $L_{i}$. $P\left(A \mid L_{i}\right)$ means probability of the accident happen while the vehicle changing the lane. By assumption that the changing point of one car uniformly distributed between, so, $P(A)$ can be easily computed .In order to reduce the probability of traffic accidents, we control the probability of collision below 0.05 . That means we have to control the distance between two vehicles. And then the density of traffic will be limited. Finally, the size range of the fan-in area can also be determined. In this way, our model will be more accurate.[3]

$$
\left\{\begin{array}{c}
P(A)=\frac{y}{d_{i}} \leq r \\
d_{i}=v_{i+1} t+\frac{v_{i+1}}{2 a_{i+1}}-\frac{v_{i+1}^{2}}{2 a_{i}}
\end{array}\right.
$$

Where " $\mathrm{r}$ " is the risk of cars collision. According to the formula ,it can limit the safe distance. It can narrow the original minimum safety distance of the vehicles. Take the three-lane and eight-tollbooth as an example. We get the graphics of the optimized fan-in areas. The probability of free lane change vehicle collision is equal to the probability in oppression lane change situation plus the probability in free lane change situation. And the same as other car oppression lane change.

Using the simulation software, according to the merger model we can obtain the vehicle driving condition diagram:

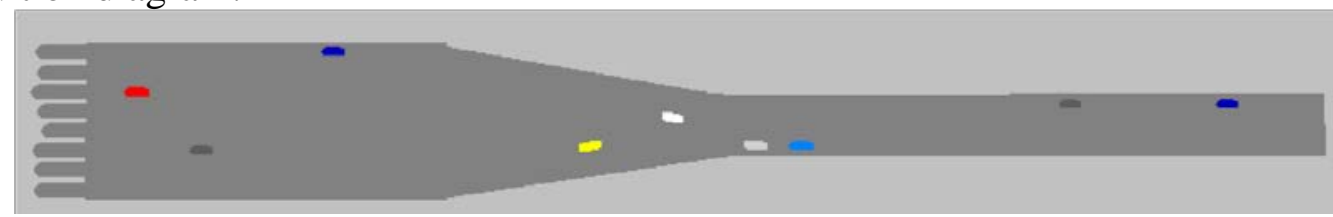

Figure 2: Vehicle driving simulation diagram

\section{Modeling the amount of different tollbooths}

There are three types of tollbooths. And the driving mode is different.They are human-staffed tollbooths, automated tollbooths and electronic toll collection booths (ETC).

We assume that the proportion of automatic vehicles less than $50 \%$. And automatic vehicles only drive into the ETC booths. Because the ETC booth's service time is very short, we placed them on the outside of the tollbooth. So that manual driving vehicles can save time to go through the toll station. In other words, the traffic congestion can be reduced.

Charge flow chart is as follows: 


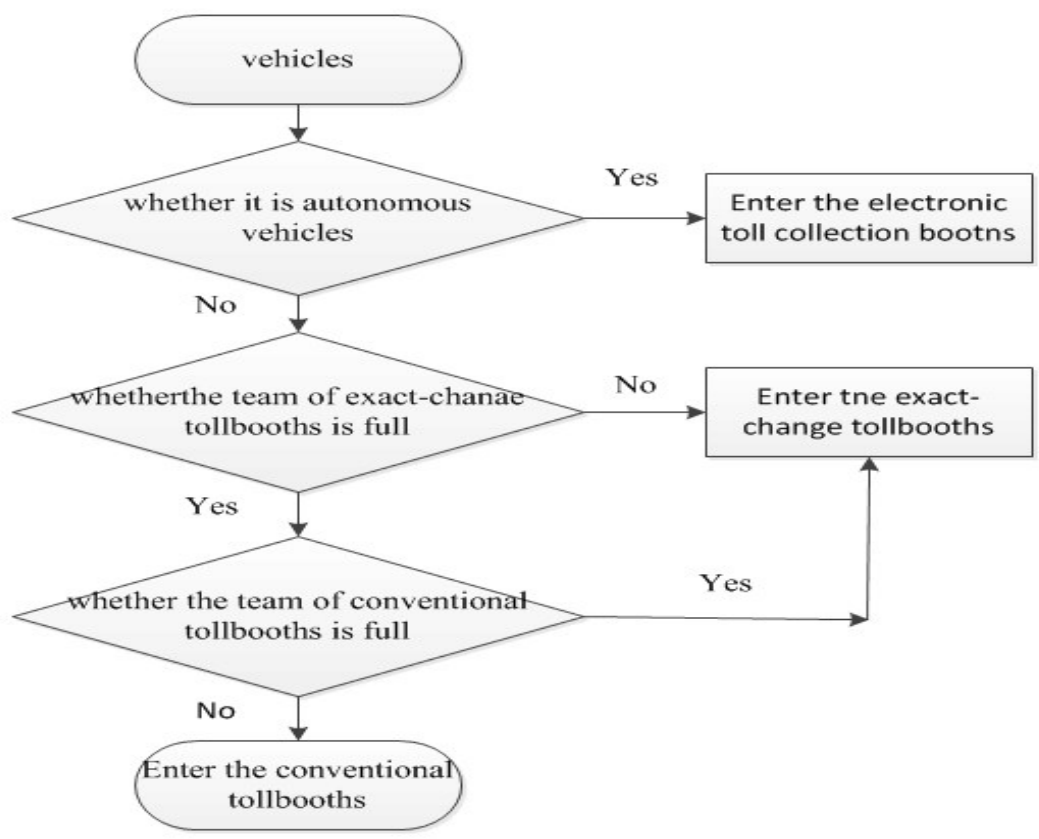

Figure 3: flow diagram

Here we use the principles of fluid mechanics and cellular automata calculation method to solve this problem. Because here we consider the situation with automatic vehicles, the speed of the vehicle will be affected by the driver's reaction time and the width of the fan-in area is affected by the service time.

\section{The Solution of Model}

We delimit the proportion of automatic vehicles as $\alpha$.And the proportion of the vehicles pass through the automated tollbooth is $\beta$, and human-staffed tollbooth is $\gamma$.According to the experience of the life, we assume driver's reaction time is $1.2 \mathrm{~s}$, and the automatic vehicle's reaction time is $0.75 \mathrm{~s}$. So, the average reaction time $t_{r a}$ :

$$
t_{r a}=\frac{0.75 \alpha+1.2(1-\alpha)}{8}
$$

The time vehicle through the human-staffed tollbooth is $10 \mathrm{~s}$, and automated tollbooth is $6 \mathrm{~s}$. The time vehicle through the electronic toll collection booths is $1 \mathrm{~s}$. So, the average service time $t_{s a}$ :

$$
t_{\text {sa }}=\frac{\alpha+6 \beta+10 \gamma}{8}
$$

The velocity of the vehicles which pass through different kinds of tollbooths are different.

Table 1:The relationship of the ratio of three kinds of tollbooths.

\begin{tabular}{|c|c|}
\hline Range of $\alpha$ & $B_{\mathrm{ETC}}: B_{\mathrm{AUTO}}: B_{\mathrm{MAN}}$ \\
\hline $0<\alpha \leq 12.5 \%$ & $1: 6: 1$ \\
\hline $12.5 \%<\alpha \leq 25 \%$ & $2: 2: 4$ \\
\hline $25 \%<\alpha \leq 37.5 \%$ & $3: 1: 4$ \\
\hline
\end{tabular}

$B_{\mathrm{ETC}}: B_{\mathrm{AUTO}}: B_{\mathrm{MAN}}$ means the proportion of the number among the human-staffed tollbooths, automated tollbooths and electronic toll collection booths.

\section{The Improvement of Model}

We call the curves which is on both sides of the fan in area transition curve. The angle between the start of the transition and the line perpendicular to the toll station is called corner and is recorded as $\theta$. 
According to the principles of tollbooth construction, the angle of the corner can't be too big. Otherwise the probability of the occurrence of traffic accidents will be great. However, considering the cost of construction, the length of the fan in area should be as small as possible. In order to save costs, we take the smallest length of the fan in area. In the case where the length of the fan-in area is at a minimum, we manually adjust the size of the corner. According to our count, the safe tangent of the corner is in the range of $1 / 7$ to $1 / 3$.

\section{Complex Distributing Wave Mode}

We define the tollbooth in $Z_{i}$ and there are totally b tollbooths. So,the serial numbers of tollbooths from left to right are $Z_{1}, Z_{2}, Z_{b}$.

We define the lane in $M_{j}$ and there are totally $m$ lanes. So,the serial numbers of tollbooths from left to right are $M_{1}, M_{2}, M_{m}$.

The driver who is in the left-most lane has a desired number and we define this number as $l$. And the desire number of the driver want to go to the lanes which is just next to his lane is $1-1$. And so on. Here we specify that there are $\mathrm{x}$ tollbooths on the left of the tollbooth which is opposite to the drive's lane. And $\mathrm{x}$ is not less than $l$ and not greater thanb.Thus, we can get a desire matrix $W$ :

$$
W=\left[\begin{array}{cc}
l-x & l-(x+1) \\
l-[b-(x+1)] & l-[b-(x+1)-1]
\end{array}\right]
$$

\section{Two Vehicle Collision}

In this problem, because more than two car collision probability is very small, here we only consider the probability of two cars crashed.

The collisionprobability of the vehicles drive from its own lane of the tollbooths to other tollbooths $Z_{i}$ is $P_{M_{j}}^{Z_{i}}$. Due to the collision probability while the vehicle change the lane $p$ :

$$
p=\frac{\text { Desire for tollbooths to transform }}{\text { Sum of desire for each tollbooth to transform }}
$$

Thus, we can obtain:

$$
P_{M_{j}}^{Z_{i}}=\sum_{p=1}^{i}\left(P_{M_{j}}^{Z_{p}} \times \sum_{q=1}^{i} P_{M_{j+1}}^{Z_{q}}\right)
$$

Through the above formula, we can calculate the collision probability of two vehicle collision. We found that, in a way, the more lane and tollbooth, the higher collision probability of two vehicle collisions is. Because the road conditions will be more complexly.

Therefore, in order to reduce the probability of collision, the number of tollbooths cannot be too much. In addition, the fan in and fan out areas should be as large as possible.

\section{Conclusion}

By developing the models, we get the optimal design of toll station. Firstly, we use the theory of fluid mechanics to obtain the relationship of the velocity and the density of vehicles. According to this, we can initially draw the shape of the fan-in area. Then, combining with the safety performance of the model, we manually adjust the model. Secondly, the merge mode of the vehicle is deduced. We mainly consider the pressure lane change situation, and obtain the collision probability of vehicles. Next, we use the principle of cellular automata and combine with the actual situation to get the best solution of how to place the different kind of tollbooths. We believe that the work we have presented here is a significant and successful attempt at solving this problem. 


\section{References}

[1] Shao Chunfu, Xiao Chongzi, Wang Bobing, MengMeng. “A Model of Velocity - density Relationship for Congested Traffic Flow under Minimum Safe Space Constraints.Journal of Traffic and Transportation Engineering,2015,(01):92-99.

[2] Ma Xiaolong, Ma Dongfang, Wang Dianhai. “Traffic Velocity-Density Relation Modeling Based on Logistic Curve.” China Journal of Highway and Transport, 2015,(04):94-100.

[3]Zhao Hantao, Mao Hongyan. "A cellular automaton model for multi - lane traffic flow with emergency vehicles.” Chinese Journal of Theoretical Physics, 2013,(06):53-60.

[4] Li Qilang. "Study on phase diagram and cellular automata model of traffic state at junction." University of Science and Technology of China, 2012.

[5] Liu Weiwei, Lei Bo, Ma Siwei, Hang Zebin. "Queuing Theory Model Analysis of Expressway Toll Station Design.” Communications Science and Technology, 2013,(04):184-185.

[6] Su Li. "Study on Design of Toll Station of Expressway.” Changan University, 2012. 